\title{
Comportamento da temperatura da pele durante uma meia maratona em ambiente quente sob radiação solar
}

https://doi.org/10.11606/issn.1981-4690.v35i3p43-50

\author{
Jefferson Fernando Coelho Rodrigues Junior* \\ Isabel Cristina de Oliveira Almeida** \\ Vicenilma de Andrade Martins s*** $^{* * *}$ \\ Francisco Railson de Oliveira Cruz ${ }^{* * *}$ \\ Jodimar Ribeiro dos Reis Junior ${ }^{* * *}$ \\ Christian Emmanuel Torres Cabido**** \\ Mario Norberto Sevilio de Oliveira Junior ${ }^{* * *}$
}

*Universidade Federal de Minas Gerais, Belo Horizonte, MG, Brasil. **Fraleon Esportes, Pará de Minas, MG, Brasil.

***Universidade

Federal do Maranhão, São Luís, MA, Brasil.

\section{Resumo}

O objetivo é avaliar a temperatura da pele (TP) em diferentes regiões durante uma meia maratona em ambiente quente sob radiação solar. $O$ estudo foi realizado em 10 corredores com idades de

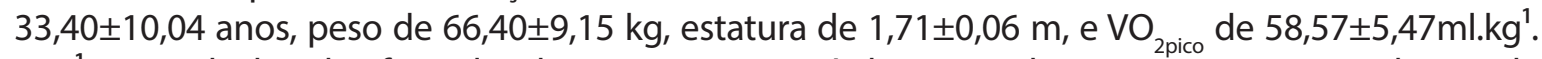
$\mathrm{min}^{1}$. A corrida de $21 \mathrm{~km}$ foi realizada em um trajeto pré-determinado, com temperatura ambiente de $27,96 \pm 1,70^{\circ} \mathrm{C}$, umidade relativa do ar 76,88 $7,49 \%$ e Índice de Temperatura de Globo e Bulbo Úmido de $25,80 \pm 1,18^{\circ} \mathrm{C}$. Medidas de TP foram mensuradas antes, a cada $3 \mathrm{~km}$ e ao término da corrida. Para todos os testes o nível foi de $\mathrm{P}<0,05$, para as comparações entre os momentos utilizou-se o teste

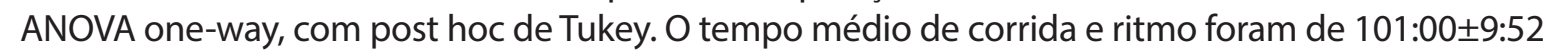
minutos e 4:48 $\pm 00: 16 \mathrm{~min} \cdot \mathrm{km}^{-1}$, respectivamente. Observou-se que a temperatura de todas as regiões da pele foi reduzida em $1,35 \pm 1,75 \mathrm{C}^{\circ}$ ao longo da corrida, a temperatura do topo da cabeça foi a menor $31,2^{\circ} \mathrm{C} \pm 2,11^{\circ} \mathrm{C}$ e a da testa foi a maior $33,7 \pm 1,35^{\circ} \mathrm{C}$. Em nosso estudo, a TP diminuiu, entretanto, a magnitude da redução é diferente, devido a particularidade de cada região avaliada, seja pelo fluxo sanguíneo, número de glândulas sudoríparas, densidade das glândulas ou pela exposição à radiação.

Palavras-chave: Corrida; Estresse fisiológico; Exercício.

\section{Introdução}

Nos últimos anos observou-se um aumento de praticantes em modalidades esportivas outdoor. Dentre essas modalidades destaca-se a corrida de rua $^{1}$, considerada em 2011 como a modalidade esportiva mais praticada no mundo. Entre os anos de 2005 e 2013, o número de provas de corrida de rua aumentou $92 \%$ e o número de participantes em $170 \%{ }^{2}$. Dentre as corridas de rua, a meia maratona $(21,1 \mathrm{~km})$ é uma das mais populares ${ }^{3}$, sendo nos Estados Unidos a prova com maior número de concluintes ${ }^{4,5}$.

Praticantes de atividade física submetidos até $18^{\circ} \mathrm{C}$ de temperatura ambiente possuem baixa possibilidade de lesôes induzidas pelo calor, atividades praticadas entre $18^{\circ} \mathrm{C}$ e $23^{\circ} \mathrm{C}$ apresentam risco moderado a saúde, entre 23 e $28^{\circ} \mathrm{C}$ as câimbras e exaustão se tornam mais frequentes, nessas situações as chances de intermação são maiores. Índices acima de $28^{\circ} \mathrm{C}$ a intermação torna-se um risco eminente ${ }^{6}$, trazendo malefícios ao organismo, podendo levar o praticante ao óbito 7 .

Para sobreviver a grande variedade climática na superfície terrestre, os seres humanos por meio de atitudes comportamentais conseguem facilitar ou dificultar a produção de calor, por meio de roupas e tecnologias ${ }^{8}$. Essas estratégias são utilizadas para 
ajudar o corpo a manter a temperatura interna dentro de uma faixa de normalidade $36^{\circ} \mathrm{C}$ e $37,5^{\circ} \mathrm{C}$. Os seres humanos são considerados homeotérmicos por conseguirem ajustar o balanço entre a produção e dissipação de calor ${ }^{10}$. A temperatura ambiente influencia a homeotermia, interferindo no bemestar, realização de tarefas ${ }^{11}$ e nas relaçôes de ganho e perda de calor. É considerado quente o ambiente com temperaturas acima de $24,1^{\circ} \mathrm{C}$, nessas condições as chances de hipertermia aumentam ${ }^{12}$ promovendo uma redução no desempenho físico ${ }^{13,14}$.

Em condiçốes climáticas em que a temperatura do ar é inferior a temperatura da pele, a dissipação de calor pelo corpo é facilitada por radiação e condução. Entretanto, quando submetidos a ambientes em que a temperatura do ar é superior à da pele, o corpo que antes dissipava calor, passa a facilitar o ganho de calor tanto por radiação como por convecção. Assim, a evaporação é o principal mecanismo de dissipaçáo de calor em ambientes quentes sendo considerada a principal defesa para o superaquecimento ${ }^{15}$. Nessas circunstâncias, tudo que impede ou dificulta a evaporação acarretará acúmulo de calor, consequentemente aumentando a temperatura interna. A produção de calor metabólico, somado a dificuldade de dissipação pode antecipar a fadiga, limitando a continuidade do exercício na mesma intensidade, além de causar câimbras, sincope e convulsóes ${ }^{16}$.

O copo humano possui um grande sistema de isolamento térmico, composto pela pele, tecidos subcutâneos e adiposos, esse sistema de isolamento ajuda a manter os órgãos internos a uma temperatura constante, mesmo quando a temperatura da pele se aproxima do ambiente ${ }^{13}$. A pele conta com uma extensa rede de vasos sanguíneos e responde a impulsos adrenérgicos e mudanças térmicas locais ${ }^{17}$, possui receptores térmicos distintos para o frio (corpúsculos de Krause) localizados nas regiôes limítrofes da pele e os receptores térmicos para o calor (corpúsculos de Ruffini) localizados nas regiôes da pele desprovidas de pelo, apesar da localização distinta ambos são localizados na derme ${ }^{6}$.

A temperatura da pele possui variaçóes, e sofre influência direta do ambiente, são em média $0,3^{\circ} \mathrm{C}$ menores que a temperatura interna. A pele é o principal responsável pela troca de calor entre o ambiente e a parte interna do corpo, ambos importantes na regulação da temperatura corporal tanto em repouso como durante o trabalho ${ }^{18}$.

Durante o exercício físico a temperatura interna é elevada, devido à produção metabólica de calor, potencializando as chances de hipertermia quando realizado no calor, podendo aumentar em até 25 vezes o metabolismo ${ }^{6}$. Quando não dissipado o calor metabólico gerado aumenta a temperatura em $1^{\circ} \mathrm{C} \mathrm{em}$ apenas 4 minutos de atividade ${ }^{19}$, isso devido ao calor ser um subproduto do metabolismo.

Considerando a popularidade da corrida de rua tanto em número de provas como em número de adeptos e concluintes em ambientes quentes, tornase evidente a necessidade de se investigar os efeitos dessas provas sob radiaçáo solar em ambiente quente nas variáveis termorregulatórias. Diante do exposto, o presente estudo teve como objetivo avaliar a temperatura da pele em diferentes pontos durante uma corrida de meia maratona em ambiente quente sob radiação solar.

\section{Método}

O estudo descritivo de delineamento observacional foi aprovado pelo Comitê de Ética em pesquisa da Universidade Federal do Maranháo sob protocolo $\mathrm{n}^{\circ} 1.548 .709 \mathrm{em}$ 17/05/2016. Após a explicação dos objetivos e procedimentos aos quais seriam submetidos todos os participantes assinaram o TCLE.

A amostra foi composta por 10 corredores amadores, do sexo masculino, residentes em Sáo Luis, Maranhão. Os dados referentes às características da amostra estâo presentes na TABELA 1.
Foram incluidos no estudo: a) praticantes de corrida por no mínimo dois anos, b) frequência semanal de treinamento mínima de cinco dias, c) ausência de lesôes osteomioarticulares nos ultimos seis meses, d) ter participado de uma meia maratona nos ultimos seis meses, e) não estar utilizando antipiréticos ou algum outro tipo de medicamento que comprometesse os resultados da pesquisa. Esses critérios foram utilizados para garantir que eles eram capazes de completar a prova em alto nível, com baixo risco. 
TABELA 1 - Caracterização da amostra dos meio-maratonistas $(n=10)$.

\begin{tabular}{lcc}
\hline Variáveis & Média \pm DP & CV $(\mathbf{9 5} \%$ IC) \\
\hline Idade (anos) & $33,4 \pm 10,04$ & $30,0(22,0-52,0)$ \\
Massa corporal $(\mathrm{kg})$ & $66,4 \pm 9,15$ & $13,79(52,4-80,8)$ \\
Estatura $(\mathrm{m})$ & $1,71 \pm 0,06$ & $3,8(1,56-1,78)$ \\
$\mathrm{IMC}\left(\mathrm{kg} / \mathrm{m}^{2}\right)$ & $22,58 \pm 2,05$ & $9,0(19,8-25,5)$ \\
$\mathrm{ASC}\left(\mathrm{m}^{2}\right)$ & $1,78 \pm 0,14$ & $8,09(1,50-1,99)$ \\
$\Sigma$ dobras cutâneas $(\mathrm{mm})$ & $72,31 \pm 33,75$ & $46,68(36,21-146,9)$ \\
$\mathrm{VO}_{\text {2pico }}\left(\mathrm{ml} . \mathrm{kg}{ }^{-1} \cdot \mathrm{min}^{-1}\right)$ & $58,75 \pm 5,47$ & $9,31(49,1-65,1)$ \\
$\%$ Massa gorda & $10,81 \pm 5,89$ & $54,4(5,3-23,3)$ \\
Quilometragem semanal $(\mathrm{km})$ & $62,5 \pm 28,80$ & $46,07(30,0-100,0)$ \\
\hline
\end{tabular}

Legenda:

DP: desvio padrão

IMC: índice de massa corporal,

ASC: área de superfície corporal,

$\Sigma$ : somatório de dobras cutâneas,

$\mathrm{VO}_{2 \text { pico }}$ : maior valor de consumo de oxigênio atingido,

CV: Coeficiente de variação,

IC: Intervalo de confiança.

\section{Instrumentos / Procedimentos}

As avaliaçóes fisiológicas foram feitas depois de 48 horas sem atividade física, seguindo a seguinte ordem: estatura, massa corporal, dobras cutâneas e o teste ergoespirométrico, para determinar o Consumo Máximo de Oxigênio.

Para a avaliaçáo da massa corporal foi utilizado a balança digital (Welmy, modelo W300, Brasil) com precisão de 0,02 gramas e capacidade de $150 \mathrm{~kg}$, a estatura foi mensurada por meio do estadiômetro portátil com escala de medida em $0,1 \mathrm{~cm}$. Além disso, o índice de massa corporal (IMC) foi calculado, através $\mathrm{da}$ fórmula peso $(\mathrm{Kg}) /$ estatura $\left(\mathrm{m}^{2}\right)^{20}$.

As dobras cutâneas foram mensuradas utilizandose um plicômetro científico (Sanny, Brasil) com sensibilidade de $0,1 \mathrm{~mm}$. O cálculo da densidade foi realizado a partir da equação de JACKSON e Pollock $^{21}$ para homens, utilizando sete dobras, sendo o percentual de gordura estimado através da equação de $\operatorname{Siri}^{22}$.

$\mathrm{O}$ teste ergoespirométrico iniciou com um aquecimento de 60 segundos, a uma intensidade de $8 \mathrm{~km} / \mathrm{h}$, em seguida o incremento foi de $1 \mathrm{~km} / \mathrm{h}$ a cada 60 segundos e em todos os estágios foi adotado uma inclinação de $1 \%$.

$\mathrm{A} \mathrm{T}_{\text {pele }}$ foi mensurada através de um termômetro infravermelho (FLUKE, 566) com mira LASER, a uma distância de $15 \mathrm{~cm}$, regulada por uma régua.
Os locais de medida da $\mathrm{T}_{\text {pele }}$ foram: $\mathrm{T}_{\text {cabeca }}$ (ponto central da cabeça), $\mathrm{T}_{\text {testa }}$ (uma polegada acima da sobrancelha, porção lateral), $\mathrm{T}_{\text {bochecha }}$ (três polegadas antes da orelha), $\mathrm{T}_{\text {peito }}$ (duas polegadas ao lado do mamilo), $\mathrm{T}_{\text {braço }}$ (na porção medial do braço, parte lateral), e $\mathrm{T}_{\text {coxa }}$ (região anterior da coxa, porção medial) todas as medidas foram padronizadas, marcadas por um pincel para quadro branco (PILOT $2 \mathrm{~mm}$ ) e coletadas ao lado direito do avaliado. As mensuraçóes ocorreram dois minutos antes do início da corrida, a cada três quilômetros e imediatamente ao térmico da corrida.

A temperatura ambiente, temperatura de globo, umidade relativa do ar e o índice IBUTG foram monitorados pelo psicrômetro Extech HT30 Heat Stress WBGT Meter, modelo Model HT30 (FLIR Comercial Systems Inc, Nashua, NH).

A meia maratona foi realizada em ambiente outdoor, sob radiação solar em um trajeto prédeterminado e sinalizado a cada 300 metros, com temperatura ambiente (TA) de $27,96 \pm 1,70^{\circ} \mathrm{C}$, temperatura de globo (TG) de $28,52 \pm 2,51^{\circ} \mathrm{C}$, umidade relativa do ar (URA) de 76,88 $\pm 7,49 \%$ e Índice de Temperatura de Globo e Bulbo Úmido (WBTG) de 25,80 $\pm 1,18^{\circ} \mathrm{C}$.

Durante a corrida teve um ponto fixo de hidratação, a cada 3,000 metros onde foi permitida 
a ingestão de água ad libitum, a quantidade de água ingerida foi monitorada para posterior correção do cálculo de sudorese total. Os voluntários completaram a prova em intensidade autorregulada.

A desidratação dos participantes foi mensurada através do percentual de desidratação, taxa de sudorese total e quantidade de água ingerida. Todos os voluntários foram pesados antes e após a meia maratona apenas com shorts, sem calçado.

Para determinar o \% de desidratação foi utilizado a equação utilizada por MotTA e Quintão ${ }^{23}$.

Equação 1: Cálculo da taxa do percentual de desidratação.

$$
\% \text { desidratação }=\frac{(\text { Peso Inicial-Peso Final })^{*} 100}{\text { PesoFinal }}
$$

O \% de perda corporal foi calculado pela diferença entre a massa corporal antes e após a prova, sendo essa diferença corrigida pela quantidade de água consumida.

Equação 2: Cálculo da perda de massa corporal em percentual.

$$
\% \text { Perda de massa corporal }=\frac{\Delta \text { Massa Corporal }}{\text { Massa corporal Inicial }} * 100
$$

\section{Resultados}

Os participantes completaram a prova simulada em 101:00 \pm 9:52 minutos em média, o ritmo de corrida médio dos voluntários durante a meia maratona foi de $4: 48 \mathrm{~min} / \mathrm{km}$ a uma intensidade de $91,81 \pm 5,80 \%$ da frequência cardíaca máxima, com média de 167,07 $\pm 12,80 \mathrm{bpm}$.

Os dados referentes a temperatura da pele dos voluntários estão presentes na TABELA 2.

Conforme mostra a TABELA 2, apesar da diferença nos valores de temperatura da pele nas diferentes regiōes avaliadas, observa-se que todos os
A taxa de sudorese total foi calculada pela diferença na massa corporal, relativizada pela área de superfície corporal dividida pelo tempo da meia maratona. Após a meia maratona os voluntários foram secos com papéis toalha e a sudorese foi corrigida pela ingestáo de água ocorrida durante a meia maratona.

Equação 3: Cálculo da taxa de sudorese total.

Taxa de sudorese total $=\frac{\Delta \text { Massa Corporal-água consumida }}{\frac{\text { ASC }}{\text { TM }}}$

Legenda:

ASC - área de superfície corporal.

$\mathrm{TM}$ - tempo em minutos.

\section{Análise estatística}

A normalidade dos dados foi previamente avaliada por meio do teste Shapiro-Wilk. Comparaçóes do momento basal, e recuperação pós-exercício foram feitas por meio do teste ANOVA one-way, com post hoc de Tuckey. Adotou-se nível de significância estatística de $\mathrm{p}<0,05$.

valores de temperatura da pele reduziram ao longo da prova de $21 \mathrm{~km}$, (topo da cabeça $-2,34 \pm 1,33 \mathrm{C}^{\circ}$, testa $-1,09 \pm 1,69 \mathrm{C}^{\circ}$, bochecha $-0,49 \pm 1,87 \mathrm{C}^{\circ}$, peito $-1,78 \pm 1,35 \mathrm{C}^{\circ}$, braço $-1,57 \pm 2,25 \mathrm{C}^{\circ}$ e coxa $\left.-0,83 \pm 1,44 \mathrm{C}^{\circ}\right)$ entretanto apenas as regióes do peito, braço e coxa apresentaram diferença $(\mathrm{p}<0,05)$.

Apesar da diferença nos valores de temperatura da pele nas diferentes regióes avaliadas durante os $21 \mathrm{~km}$, a temperatura do topo da cabeça foi a menor $\left(31,0^{\circ} \mathrm{C} \pm 2,05^{\circ} \mathrm{C}\right)$ e a da testa foi a maior $\left(33,6 \pm 1,33^{\circ} \mathrm{C}\right)$. 
TABELA 2 - Comportamento da temperatura da pele durante a meia maratona.

\begin{tabular}{|c|c|c|c|c|c|c|c|c|}
\hline & Repouso & $3 \mathrm{~km}$ & $6 \mathrm{~km}$ & $9 \mathrm{~km}$ & $12 \mathrm{~km}$ & $15 \mathrm{~km}$ & $18 \mathrm{~km}$ & $21 \mathrm{~km}$ \\
\hline & $\left({ }^{\circ} \mathrm{C}\right)$ & $\left({ }^{\circ} \mathrm{C}\right)$ & $\left({ }^{\circ} \mathrm{C}\right)$ & $\left({ }^{\circ} \mathrm{C}\right)$ & $\left({ }^{\circ} \mathrm{C}\right)$ & $\left({ }^{\circ} \mathrm{C}\right)$ & $\left({ }^{\circ} \mathrm{C}\right)$ & $\left({ }^{\circ} \mathrm{C}\right)$ \\
\hline Topo da cabeça & $32,8 \pm 1,9$ & $31,7 \pm 2,6$ & $31,7 \pm 2,6$ & $31,1 \pm 2,1$ & $31,0 \pm 2,3$ & $30,2 \pm 1,3$ & $30,8 \pm 1,8$ & $30,4 \pm 1,5$ \\
\hline Testa & $34,7 \pm 1,1$ & $33,5 \pm 1,0$ & $33,7 \pm 1,6$ & $33,8 \pm 0,9$ & $33,6 \pm 2,1$ & $33,4 \pm 1,5$ & $33,4 \pm 1,0$ & $33,6 \pm 1,2$ \\
\hline Bochecha & $33,6 \pm 1,3$ & $33,8 \pm 1,9$ & $33,4 \pm 1,8$ & $34,1 \pm 1,2$ & $34,0 \pm 1,6$ & $33,8 \pm 2,2$ & $33,2 \pm 1,7$ & $33,1 \pm 1,7$ \\
\hline Peito & $33,4 \pm 1,8$ & $32,1 \pm 1,1$ & $32,4 \pm 1,7$ & $32,1 \pm 0,6$ & $32,3 \pm 1,5$ & $32,4 \pm 1,5$ & $31,5 \pm 1,9$ & $31,6 \pm 1,0^{*}$ \\
\hline Braço & $33,5 \pm 2,1$ & $32,0 \pm 1,8$ & $32,6 \pm 1,3$ & $32,3 \pm 1,5$ & $32,2 \pm 1,0$ & $32,3 \pm 1,1$ & $31,8 \pm 1,9^{*}$ & $31,9 \pm 1,4^{*}$ \\
\hline Coxa & $32,9 \pm 1,4$ & $32,0 \pm 1,6$ & $32,7 \pm 1,9$ & $32,5 \pm 1,2$ & $32,5 \pm 1,2$ & $32,3 \pm 1,5^{*}$ & $32,4 \pm 1,6$ & $32,1 \pm 1,6^{*}$ \\
\hline Média & $33,4 \pm 1,6$ & $32,5 \pm 1,8^{*}$ & $32,7 \pm 1,9^{*}$ & $32,6 \pm 1,7^{*}$ & $32,5 \pm 1,8^{*}$ & $32,4 \pm 1,8^{*}$ & $32,1 \pm 1,8^{*}$ & $32,1 \pm 1,6^{*}$ \\
\hline
\end{tabular}

Comportamento da temperatura da pele em diferentes pontos do corpo durante a meia maratona.

Os dados estão apresentados em média e desvio padrão. *diferenças significativas se comparado aos valores de repouso $(p<0,05)$

${ }^{\circ} \mathrm{C}$ - temperatura em Célsius.

\section{Discussão}

Este estudo teve como objetivo avaliar a temperatura da pele em diferentes pontos durante uma meia maratona realizada ambiente quente sob radiação solar. De acordo com nossos achados corredores submetidos a uma meia maratona em ambiente quente, obtiveram redução da temperatura da pele já nos primeiros três quilômetros. A temperatura da pele no início da corrida foi de $33,4 \pm 1,6^{\circ} \mathrm{C}$, atingindo $32,1 \pm 1,6^{\circ} \mathrm{C}$ ao final da prova, apresentando um acréscimo de 1,4 \pm $1,7^{\circ} \mathrm{C}$ no decorrer da prova, sendo essa redução significativa a partir do terceiro quilômetro. Isso pode ser explicado devido as condiçóes ambientais (TA $27,96 \pm 1,69^{\circ} \mathrm{C}$ e URA $76,88 \pm 7,49 \%$ ) com diferença de $\left(4,6 \pm 1,7^{\circ} \mathrm{C}\right)$ entre a temperatura da pele e do ambiente. Essas condiçóes ambientais somadas a uma maior requisição muscular gera um aumento da temperatura central ${ }^{24-25}$

$\mathrm{O}$ aumento da temperatura central durante o exercício ocorre devido a estimulaçáo do hipotálamo-hipófise-adrenal aumentando a secreção do córtex adrenal, estimulando a gliconeogênese e a lipólise (metabolismo) contribuindo para aumentar a termogênese. Esse aumento é detectado pelos termorreceptores centrais e da pele, sendo processadas pela regiáo pré-óptica do hipotálamo para estimular a dissipação do calor metabólico ${ }^{26-27}$.
Devido a amostra ser composta por indivíduos treinados $\left(58,75 \pm 5,47 \mathrm{ml} \cdot \mathrm{kg}^{-1} \cdot \mathrm{min}^{-1}, 62,5 \pm 28,80\right.$ $\mathrm{km} / \mathrm{sem}$ ) e nativos da regiáo tropical, provavelmente possuem um maior volume plasmático, uma maior conservação de sódio ${ }^{28}$ e uma antecipaçáo do ponto de produção de suor se comparado a congêneres destreinados náo aclimatados, facilitando o resfriamento da pele e consequentemente a perda de calor por evaporaçãa ${ }^{29}$ além de um aumento do fluxo sanguíneo periférico ${ }^{30}$ e vasodilatação cutânea ${ }^{31-32}$ aumentando a tolerância ao calor ${ }^{24}$.

Durante o exercício no calor o aumento da sudorese ocorre devido um maior recrutamento de glândulas sudoríparas, posteriormente esse aumento é dado pelo aumento no volume de suor secretado por cada glândula ${ }^{33}$ isso explica as diferenças de temperatura nos pontos avaliados. A temperatura média da testa durante a meia maratona foi de 33,6 $\pm 1,33^{\circ} \mathrm{C}$ enquanto a temperatura da coxa foi de $32,4 \pm 1,60^{\circ} \mathrm{C}$, apresentando uma diferença de 1,2 $\pm 1,5^{\circ} \mathrm{C}$ entre as regióes. Isso pode ser explicado pela região da testa apresentar uma grande exposição à radiação solar.

Apesar da testa apresentar maiores valores de temperatura $\left(1,33 \pm 1,86^{\circ} \mathrm{C}\right)$ em comparação as demais regióes, a regiáo da testa apresenta uma taxa de sudorese entre 25 e $100 \%$ maior que a região da 
$\operatorname{coxa}^{34}$, devido a testa ser a região que apresenta um maior número de glândulas sudoríparas ativadas. Além disso, a região da testa apresenta glândulas sudoríparas com maior densidade ${ }^{35}$.

A temperatura média do topo da cabeça no início da corrida foi de $32,8 \pm 1,9^{\circ} \mathrm{C}$ reduzindo $1,1 \pm 2,3^{\circ} \mathrm{C}$ nos primeiros três quilômetros, chegando aos $30,4 \pm$ $1,5^{\circ} \mathrm{C}$ ao final da prova, apresentando um delta de 2,3 $\pm 1,3^{\circ} \mathrm{C}$. Vale destacar que a temperatura do topo da cabeça foi a menor temperatura em todos os momentos se comparado as outras regióes. Isso pode ser explicado pela região da cabeça ser responsável pela maior parte da dissipação de calor no corpo, além de contar com a barreira natural do couro cabeludo.

Em resposta ao aquecimento central do organismo como forma de controlar a temperatura, o corpo aumenta a taxa de sudorese durante a exposição a ambientes em que a temperatura da pele se encontra abaixo da temperatura ambiente ${ }^{36}$ com isso os participantes da pesquisa tiveram uma desidratação média de 3,40 $\pm 0,98 \%$, que representa $3,76 \pm 0,82$ $\%$ da massa corporal pré exercício.

\section{Conclusão}

Durante a corrida outdoor de $21 \mathrm{~km} \mathrm{em}$ ambiente quente e úmido, a temperatura da pele diminuiu nos primeiros três quilômetros, permanecendo menor se comparado aos valores basais. Entretanto a magnitude de redução da temperatura da pele é diferente, devido a particularidade cada regiáo seja pelo fluxo
Após a meia maratona os voluntários apresentaram uma taxa de sudorese total de $12,91 \pm 3,43 \mathrm{ml} / \mathrm{min}$, o que representa em média $774,6 \mathrm{ml} / \mathrm{h}$. Segundo a Sociedade Brasileira de Medicina Esportiva a taxa de sudorese durante atividades realizadas no ambiente quente pode variar entre $500 \mathrm{a} 2.000 \mathrm{ml} / \mathrm{h}^{36}$. A variação na taxa de sudorese pode modificar de acordo com o nível de atividade física do praticante, aclimatação, sexo e composição corporal ${ }^{24}$.

Durante a meia maratona os voluntários consumiram em média $211 \pm 215,40 \mathrm{ml}$ de água, com consumo variando entre 0 e $600 \mathrm{ml}$, vale destacar que a necessidade de tomar água varia individualmente por uma série de fatores, ambientais, nível de treinamento, composição corporal, intensidade e duração da atividade ${ }^{36}$.

Com relação às possíveis limitações, nosso estudo teve como objetivo realizar o estudo em ambiente outdoor, entretanto devido as condiçóes climáticas da cidade náo ter clima frio ou neutro, impossibilitou a comparação dessas respostas com outro ambiente.

sanguíneo da região, número de glândulas sudoríparas, pela densidade das glândulas ou pela exposição à radiação.

Diante do aumento do número de corridas de rua incluindo a meia maratona, sugerimos que sejam feitos mais estudos, em outros ambientes, para compreendermos melhor essas respostas.

\section{Conflito de interesses}

Os autores declaram não haver conflito de interesse. 


\begin{abstract}
Behavior of skin temperature during a half-marathon in a hot environment under solar radiation.

The objective is to evaluate the skin temperature (ST) in different regions during a half-marathon in a hot environment under solar radiation. The study was carried out in 10 runners aged $33.40 \pm 10.04$ years, weight of $66.40 \pm 9.15 \mathrm{~kg}$, height of $1.71 \pm 0.06 \mathrm{~m}$, and $\mathrm{VO}_{2 \text { peak }}$ of $58.57 \pm 5,47 \mathrm{~mL} . \mathrm{kg}^{1} . \mathrm{min}^{1}$. The $21 \mathrm{~km}$ run was carried out on a predetermined route, with an ambient temperature of $27.96 \pm 1.70^{\circ} \mathrm{C}$, relative air humidity $76.88 \pm 7.49 \%$ and Globe and Wet Bulb Temperature Index of $25.80 \pm 1.18^{\circ} \mathrm{C}$. The ST measurements were measured before, every $3 \mathrm{~km}$ and at the end of the race. For all tests, the level was $\mathrm{P}<0.05$, for comparisons between moments, the one-way ANOVA test was used, with Tukey's post hoc. The average running time and pace were 101:00 \pm 9: 52 minutes and 4: $48 \pm 00: 16 \mathrm{~min} \cdot \mathrm{km}^{-1}$, respectively. It was observed that the temperature of all skin regions was reduced by $1.35 \pm 1.75^{\circ} \mathrm{C}$ throughout the race, the temperature of the top of the head was the lowest $31.2^{\circ} \mathrm{C} \pm 2.11^{\circ} \mathrm{C}$ and that of the forehead was the highest $33.7 \pm 1.35^{\circ} \mathrm{C}$. In our study, the ST decreased, however, the magnitude of the reduction is different, due to the particularity of each region assessed, either by blood flow, number of sweat glands, density of the glands or by exposure to radiation.
\end{abstract}

KEYwords: Race; Physiological stress; Exercise.

\title{
Referências
}

1. Cardoso AF, Ferreira ES, Dos Santos LV. Frequência do consumo alimentar de praticantes de corrida categoria amador na cidade de Teresina-PI. Rev Bras Nutrição Esportiva. 2019;13(78):139-149.

2. Campos TM, De Moraes MB, LIMA E. Um estudo sobre a caracterizaçấo dos empreendedores das redes ligados aos circuitos de corrida de rua. Latin Am J Business Management. 2015;6(3).

3. Maioral RF. Identificação e avaliação dos atributos que influenciam a decisão de participação em eventos de corrida pedestre no Brasil. 2014.

4. Purim K, Titski A, Leite N. Hábitos solares, queimaduras e fotoproteção em atletas de meia maratona. Rev Bras Atividade Fís Saúde. 2013;18(5):636.

5. Running USA. Statistics, 2014. Disponivel em:<http://www.runningusa.org/statistics>. Acesso em: 07 abr. 2020.

6. Mcardle DM, Kacth FI, Katch VL. Nutrição para o desporto e o exercício. Rio de Janeiro, Guanabara, 2016.

7. Silami-Garcia E, Rodrigues LOC. Hipertermia durante a prática de exercícios físicos: riscos, sintomas e tratamento. Rev Bras Ciênc Esporte. 2010;19(3).

8. Mundel T, Bunn SJ, Hooper PL, Jones DA. The effects of face cooling during hyperthermic exercise in man: evidence for a integrated thermal, neuroendocrine and behavioral response. Exp Physiol. 2007;92:187-95.

9. Romanovsky AA. The thermoregulation system and how it works. In: Handbook of clinical neurology. Amsterdá, Elsevier, 2018. p. 3-43.

10. Sessler DI. Mild perioperative hypothermia. N Eng J Med. 1997;336:1730-1737.

11. Miguel AS. Manual de Higiene e Segurança do Trabalho. 12a Edição. Porto Editora. 2012.Minas Gerais, 2008.

12. De Castro Pinto KM, Rodrigues DLOC, De Paula Viveiros D J, Silami-Garcia, E. Efeitos da temperatura da água ingerida sobre a fadiga durante o exercício em ambiente termoneutro. Rev Paul Educ Fís. 2001;15(1):45-54.

13. Romanovsky AA. Skin temperature: its role in thermoregulation. Acta Physiol. 2014;210(3,):498-507.

14. Niedermann R, Wyss E, Annaheim S, Psikuta A, Davey S, Rossi, RM. Prediction of human core body temperature using non-invasive measurement methods. Int J Biometeorology. 2014;58(1):7-15.

15. Tarini VA, Vilas L, Zanuto R, Silva HC, Oliveira AS. Calor, exercício físico e hipertermia: epidemiologia, etiopatogenia, complicaçóes, fatores de risco, intervençóes e prevenção. Rev Neurociências. 2006;14(3):144-152.

16. De Melo-Marins D, Souza-Silva AA, Silami-Garcia E, Laitano O. Termorregulação e equilíbrio hídrico no exercício: aspectos atuais e recomendações. Rev Bras Ciênc Movimento. 2017;25(3):181.

17.Gagge A, Pharo G, Richard R. Mechanisms of heat exchange: biophysics and physiology. Handbook of physiology,

Rev Bras Educ Fís Esporte, (São Paulo) 2021 Jul-Set;35(3):43-50 • 49 
section. 1996. v. 4, p. 45-84.

18. Kenny GP, Journeay WS. Human thermoregulation: separating thermal and nonthermal effects on heat loss. Front Biosci. 2010;15(25990.2).

19. Veltmeijer MT, Veeneman D, Bongers CC, Netea MG, Van Der Meer JW, et al. The impact of central and peripheral cyclooxygenase enzyme inhibition on exercise-induced elevations in core body temperature. Int J Sports Physiol Performance. 2017;12(5):662-7.

20. Charro MA, Bacurau RFP, Navarro F, Pontes Junior FL. Manual de avaliação física. São Paulo: Phorte, 2010.

21. Jackson AS, Pollock ML. Generalized equations for predicting body density of men. British J Nutrition. 1978;40(3):497504.

22. Siri WE. Body composition from fluid space and density. In: Brozek J, Hanschel A, editores. Techniques for measuring body composition. Washington DC, National Academy of Science. p. 223-224, 1961.

23. Motta AMS, Quintão DF. Nível de desidratação e estratégias nutricionais utilizadas antes e durante o treino de Futebol de um grupo de adolescentes de Espera Feliz-MG. Rev Bras Nutrição Esportiva. 2016;10(59):518-23.

24. Mora-Rodriguez R. Influence of aerobic fitness on thermoregulation during exercise in the heat. Exerc Sport Scienc Rev. 2012;40(2):79-87.

25. Teunissen LPJ, De Haan A, De Koning JJ, Daanen HAM. Telemetry pill versus rectal and esophageal temperature during extreme rates of exercise-induced core temperature change. Physiol Measurement. 2012;33(6):915.

26. Taylor Nigel AS, Tipton Michael J, Kenny Glen P. Considerations for the measurement of core, skin and mean body temperatures. J Thermal Biol. 2014;46:72-101.

27. Mack GW, Nadel ER. Body fluid balance during heat stress in humans. In: Terjung R. editores. Comprehensive physiology. 2011.

28. Buono MJ, Ball KD, Kolkhorst, FW. Sodium ion concentration vs. sweat rate relationship in humans. J Appl Physiol. 2007;103(3):990-994.

29. Kenny GP, Wilson TE, Flouris AD, Fujii N. Heat exhaustion. In: Handbook of clinical neurology. 2018. Elsevier. p. 505-529.

30. Heinonen, I, Brothers, RM, Kemppainen, J, Knuuti, J, Kalliokoski KK Crandall CG. Local heating, but not indirect whole body heating, increases human skeletal muscle blood flow. J Appl Physiol. 2011;111(3):818-824.

31. Johnson JM, Minson CT, Kellogg Junior DL. Cutaneous vasodilator and vasoconstrictor mechanisms in temperature regulation. Compr Physiol. 2014;4:33-89.

32. Takeda H, Kondo S. Immunohistochemical study of angiotensin receptors in normal human sweat glands and eccrine poroma. British J Dermatol. 2001;144(6):1189-1192.

33. Shamsuddin AKM., Kuwahara T, Oue A, Nomura C, Koga S, Inoue Y, Kondo N. Effect of skin temperature on the ion reabsorption capacity of sweat glands during exercise in humans. Eur J Appl Physiol. 2005;94(4):442-447.

34. Baker LB. Sweating rate and sweat sodium concentration in athletes: a review of methodology and intra/interindividual variability. Sports Med. 2017;47(1):111-128.

35. Buono MJ, Connolly Kevin P. Increases in sweat rate during exercise: gland recruitment versus output per gland. J Thermal Biol. 1992;17(4-5):267-270.

36. Carvalho, T, Mara LS. Hidratação e nutrição no esporte. Rev Bras Med Esporte. 2010;16(2):144-148. 